1 Decreased tibial nerve movement in patients with failed back surgery syndrome

2 and persistent leg pain.

3

4 Shum $\mathrm{G}^{1,2}$, Cinnamond $\mathrm{S}^{1}$, Hutton $\mathrm{M}^{2}$, Chan $\mathrm{D}^{2}$, Chauhan $\mathrm{R}^{2}$, Bloxham $\mathrm{S}^{1}$, Choy $\mathrm{S}^{1}$,

$5 \quad$ Cheung $\mathrm{R}^{3}$ and Eldabe $\mathrm{S}^{4}$ and Clarke $\mathrm{A}^{2}$

$6{ }^{1}$ School of Sport, Health and Wellbeing, Plymouth Marjon University, United

7 Kingdom.

$8 \quad{ }^{2}$ Exeter Spinal Unit, Princess Elizabeth Orthopaedic Centre, Royal Devon and Exeter

$9 \quad$ NHS Foundation Trust, United Kingdom.

$10{ }^{3}$ Department of Rehabilitation Sciences, the Hong Kong Polytechnic University, Hong

11 Kong

$12{ }^{4}$ Department of Anaesthesia, South Tees Hospitals NHS Foundation Trust, United 13 Kingdom.

14

15

16 Corresponding author:

17 Professor Gary Shum

18 Professor of Biomechanics and Rehabilitation

19 Plymouth Marjon University

20 Plymouth PL6 8BH

21 United Kingdom

22 email: gshum@marjon.ac.uk

$23 \quad$ Tel: +441752636700 (Ext. 5310)

24 Website: https://www.marjon.ac.uk/ 
1 Source of funding:

2 The Royal Devon and Exeter NHS Foundation Trust, United Kingdom (13/SW/0029

3 and $14 / \mathrm{NW} / 0110)$.

5 Decreased tibial nerve movement in patients with failed back surgery syndrome.

7 Abstract

8 Purpose To measure and compare the total and normalised tibial nerve movement

9 during forward bending in patients with and without Failed Back Surgery

10 Syndrome(FBSS) and persistent leg pain following anatomically successful lumbar

11 decompression surgery and demonstrated no psychological stress. Nerve 12 pathomechanics may contribute to FBSS with persistent leg pain following 13 anatomically successful lumbar decompression surgery.

14 Methods Tibial nerve movement during forward bending was measured in two groups 15 of patients following anatomically successful lumbar decompression surgery. FBSS 16 group( $\mathrm{N}=37)$ consisted of patients with persistent leg pain following lumbar surgery 17 and non-FBSS(N=37) were patients with no remaining leg pain following lumbar 18 surgery. Total and normalised tibial nerve movement at the popliteal fossa was 19 measured by a previously validated ultrasound imaging technique and compared 20 between the two groups, and also between the painful and non-painful leg within the 21 FBSS group.

22 Results Both the mean total and normalised tibial nerve movement were significantly 23 decreased in the FBSS group in both legs when compared to the non-FBSS group $24(\mathrm{P}<0.05)$. The total and normalised tibial nerve movement was also more restricted in 25 the painful leg $(\mathrm{P}<0.05)$ when compared to the non-painful side within the FBSS group. 
1 Conclusion This was the first study to quantify the decreased total and normalised

2 tibial nerve mobility in FBSS patients with persistent leg pain when compared with

3 non-FBSS patients following anatomically successful lumbar decompression surgery.

4 Further research could investigate the efficacy of intervention, such as nerve

5 mobilisation in this particular group of patients with failed back surgery syndrome and

$6 \quad$ limited nerve mobility.

7

8 Word count: 249

9

10 Keywords:

11 Failed back surgery syndrome; neuropathic pain; sciatica; nerve movement; post-

12 operative pain.

13

14

15 
Introduction

2 Lumbar surgery is performed on approximately 23,592 patients each year in the United

3 Kingdom[1]. However, it is estimated that $10-40 \%$ of these patients will continue to

4 experience pre-surgical symptoms and pain despite anatomically successful surgery for

5 either lumbar intervertebral disc disorder[2] or lateral recess syndrome[3]; a condition

6 referred to as Failed Back Surgery Syndrome (FBSS)[4]. A recent study estimated the

7 incidence of FBSS at 20.8\% within 2 years of lumbar surgery[1], although incidence

8 levels vary across surgical procedures, with rates of $35-36.2 \%$ reported following

9 lumbar decompression[5] and a rate of 20-25\% reported following lumbar

10 microdisectomy[6]. FBSS results in continued pain, functional limitations and reduced

11 ability to work[1], with FBSS patients with persistent leg pain reporting much lower

12 health-related quality of life scores(EQ-5D scores of $0.16 \pm 0.3)$ than other causes of

13 neuropathic pain[4]. FBSS results in significantly increased post-surgical healthcare

14 costs that are estimated to be over $50 \%$ greater than lumbar surgery patients with no

15 continued pain[1]. Furthermore, FBSS exacts a high societal cost with up to $15 \%$ of

16 young, active participants failing to return to work despite having no overt re-herniation

17 or lumbar pathology post-microdiscectomy[7].

19 Nerve root impingement resulting in peripheral nerve pain is a common characteristic

20 of both intervertebral disc disorder and lateral recess syndrome. Peripheral nerves such

21 as the tibial nerve must bend, stretch and glide along their length within the nerve tissue

22 bed to accommodate movement of the adjacent joints whilst maintaining the

23 transmission of electrical impulses[8]. Nerve root impingement could compromise the

24 ability of a peripheral nerve to stretch and glide causing reduced neural mobility and

25 subsequent increased neural tension and associated loss of function, pain or neural 
1 fibrosis[9], increased mechanosensitivity[10], a reduction in nerve conduction,

2 inhibited axonal transportation and neural oedema[11].

4 An innovative technique was developed to measure tibial nerve movement at the knee

5 during forward bending movement of the spine[12]. During forward bending, a mean

6 tibial nerve movement of $12.2 \pm 2.2 \mathrm{~mm}$ measured at the popliteal fossa was found in

7 asymptomatic participants, which has been shown to be a reliable measurement[12].

8 The substantial proximal movement of the tibial nerve during forward bending is

9 consistent with the requirement of the nerve tract to accommodate increases in the nerve

10 bed length evoked by hip and lumbar spine flexion [12]. It was hypothesized that

11 reduced nerve movement could potentially contribute to the persistent leg pain of FBSS.

13 The aim of this study was to compare both the total and normalised tibial nerve

14 movement between FBSS and non-FBSS patients following lumbar surgery, and

15 between the painful and non-painful leg within the FBSS group. It was hypothesised

16 that people with FBSS and persistent leg pain will present with reduced total and

17 normalised tibial nerve movement when compared to people without persistent leg pain

18 following lumbar surgery. A second hypothesis was that there would be significant

19 differences in the tibial nerve movement between the painful and non-painful side in 20 people with FBSS. 
1 Materials and Methods:

2 Ethical Approval

3 Ethical approval was granted by the National Health Service (NHS) Health Research

4 Authority, United Kingdom. A total of seventy-four patients with and without post-

5 operative leg pain following discectomy or lumbar decompression were recruited.

7 Sample Size

8 Our previous work has shown that during forward bending in 24 asymptomatic

9 participants the sciatic nerve moves at the popliteal fossa by $12.2 \pm 2.2 \mathrm{~mm}[12]$.

10 However, no data regarding nerve movement in symptomatic participants has been

11 previously published. Based on a $15 \%$ difference in the tibial nerve movement between

12 the FBSS and non-FBSS group following lumbar surgery and the observed standard

13 deviation of $2.2 \mathrm{~mm}$ at $95 \%$ power and $5 \%$ alpha, 32 participants per group was required

14 in this study.

15

16 Participants

17 People with $(\mathrm{N}=37)$ or without $(\mathrm{N}=37)$ postoperative residual leg pain following lumbar

18 discectomy or decompression completed this study with the following eligibility

19 criteria:

20 Inclusion criteria:

21

22 Patients aged 18-80 years who underwent lumbar microdiscectomy or single level

23 lumbar decompression surgery, 6-12months post-operation.

24 Patients who have persistent postoperative residual leg pain as defined by: 
a) The severity of leg pain score being 5 or more on Numerical Rating Scale of Pain[13].

b) less than 5 points improvement in the Global Rating of Change Scale, in which a clinically important improvement is defined as 5 or more[14].

c) a positive straight leg raise (SLR) sign (specified as $65^{\circ}$ or less movement of the straight leg relative to the longitudinal axis of the trunk) that the test reproduced unilateral symptoms in the tested leg[15].

\section{Exclusion Criteria:}

Participants were excluded if they suffered from long standing ischaemic neuritis or any other surgery-related complications (e.g. inadequate decompression, postoperative instability, neural injury) as they may lead to postoperative residual leg pain. Patients were also excluded if identified as at risk by the Distress and Risk Assessment Method(modified Zung score $\geq 17$ and/or Modified Somatic Perception Questionnaire score $<12)[16]$, which has been shown to be an accurate assessment tool of psychological disturbance in patients with low back pain[16].

Seventy-four patients were recruited for the study in accordance with the eligibility criteria. Participants were divided into two groups dependent on surgical outcomes:

Non-FBSS group (N=37): Participants with no or minimal residual leg pain during forward bending; defined as (A) greater than 50\% improvement three months after the operation, and (B) a negative straight leg raise sign when the maximum angle between

4 the straight leg and the longitudinal axis of the trunk is $66^{\circ}$ or more[17]. 
$1 \quad$ FBSS group $(\mathrm{N}=37)$ : Participants with post-operative residual leg pain during forward

2 bending; defined as (A) either unchanged or less than 50\% improvement three months

3 after operation as defined on a visual analogue scale; and (B) a positive straight leg

4 raise sign when the maximum angle between the straight leg and the longitudinal axis

5 of the trunk is $65^{\circ}$ or less, with unilateral symptoms reproduced in the tested leg[17].

7 Subjects were assessed at the Spinal Unit of a local hospital on one occasion, three to

8 nine-months post-surgery with the following outcome measures:

9 Ultrasound recordings of linear arrays centre frequency at 7.5Mhz (Model: HL5-9ED,

10 Medison Co., Ltd, Seoul, South Korea) during forward bending were taken behind the

11 knee region in order to track movement of the tibial nerve using a similar technique

12 developed from previous research[12, 18]. The image sequences of the diagnostic

13 ultrasound cine-loops were analysed using a frame-by-frame normalised cross-

14 correlation approach implemented in MATLAB (MathWorks, Natwick, MA, USA)

15 [12]. The tracking programme used a pattern-matching algorithm based on the

16 greyscale pattern present in each of the selected region of interests to find the best match

17 region of interests in sequential frames. Displacement of the nerve in the longitudinal

18 (lateral) and axial (deep/superficial) dimensions were registered for each frame-by-

19 frame matching comparison. The programme then calculated the hypotenuse excursion

20 from the vector combination of longitudinal and axial movement.

22 Spinal and hip movements were measured within physiological ranges. Lumbar spine

23 and hip movement and coordination were measured using the three-dimensional inertia

24 measurement unit (ProMove 3D, Inertia Technology, The Netherlands) during the

25 forward bending movement. From an erect standing position, participants were 
1 instructed to bend forward as far as comfortably possible at their natural and controlled

2 pace to reach their maximum forward bend angle. The erect start position had the

3 participant standing tall, looking straight ahead with arms folded across their chest, feet

4 positioned shoulder width apart. Three bending forward movements were recorded with

5 a rest period of 2 min between each movement[12]. Markers were placed on 4

6 standardised landmarks on the posterior thigh, sacrum and L1 spinous process. Signals

7 were Analog to Digital converted (200Hz sampling frequency) and stored for offline 8 analysis.

10 The tibial nerve movement(displacement) was then normalised by dividing it by the

11 sum of the lumbar spine and hip flexion angles during each forward bending movement:

12 Normalised tibial nerve movement $\left(\mathrm{mm} /{ }^{\circ}\right)=$ Tibial nerve movement $(\mathrm{mm}) /$ Total 13 lumbar spine and hip flexion angle $\left(^{\circ}\right)$

14 To minimize bias, the researcher was blinded to each participant's information or 15 grouping during offline data analysis.

18 The improvement between before and after surgical intervention was measured with 19 the Global Rating of Change Scale with a 15-point scale (-7 to +7$)$, in which a clinically 20 important improvement was defined as 5 or more[14]. Participants were asked to rate 21 their severity of back pain and leg pain using a simple $10 \mathrm{~cm}$ visual analogue scale[19].

22 A standardized passive straight leg raise (SLR) test was performed and the maximum 23 angle between the straight leg and the longitudinal axis of the trunk measured using an 24 inclinometer. SLR sign was considered to be positive if the lift angle was $65^{\circ}$ or less, 25 with unilateral symptoms reproduced in the tested leg[17]. 
1 The level of psychological stress was measured by the Distress and Risk Assessment

2 Method[16], which is a combination of the Modified Zung depression scale and the

3 Modified Somatic Perception Questionnaire to assess depression and somatisation of

4 anxiety. The threshold scores in the Distress and Risk Assessment Method has been

5 validated in identifying psychological disturbance in patients with low back pain[16].

\section{Statistical analyses}

10 Descriptive statistics were produced of the mean and standard deviation of the angle of 11 SLR and longitudinal, axial and hypotenuse nerve excursion magnitude. Statistical 12 analysis was performed with SPSS software (Version 22.0). Intra-class correlation 13 coefficient $\left(\mathrm{ICC}_{3, \mathrm{k}}\right)$ with $95 \%$ confidence interval was calculated to determine intra14 rater reliability of the three repeat measures of forward bending.

15 The Kolmogorov-Smirnov test $(\mathrm{p}=0.065)$ concluded that the data is homogeneous, 16 parametric statistical tests were therefore conducted with the level of significance set 17 at 0.05 .

$18 \mathrm{~T}$ tests were used to compare the statistical differences in the tibial nerve movement 19 between FBSS and non-FBSS group of the painful side and non-painful side. T-tests 20 were also used to compare the clinic outcome measures between the non-FBSS and 21 FBSS groups.

23 Paired T-test was used to compare the tibial nerve movement between the painful leg 24 and the non-painful leg within each group (FBSS or Non-FBSS). 


\section{$1 \quad$ Results}

2 Subject characteristics were presented in table 1. No participants dropped out of the 3 study during the one-off assessment.

4 There were no significant differences in the scores of the Modified Zung depression

5 scale and Modified Somatic Perception Questionnaire between non-FBSS group and

$6 \quad$ FBSS group. All participant have a modified Zung depression score less than 17 and 7 no participant has a Modified Somatic Perception Questionnaire score less than 12.

8 The angle of SLR was significantly more limited in FBSS group $\left(42.0^{\circ} \pm 16.2^{\circ}\right)$ when

9 compared to non-FBSS group $\left(76.5^{\circ} \pm 7.0^{\circ}\right)$ (Table $\left.1, \mathrm{P}<0.05\right)$.

10 During the forward bending movement, the flexion range of movement of the lumbar 11 spine and hip were significantly reduced in FBSS group when compared with the non12 FBSS group (Table 1, $\mathrm{P}<0.05)$.

14 The mean $\mathrm{ICC}_{3, \mathrm{k}}$ for measuring the normalised longitudinal, axial and hypotenuse 15 movement of the tibial branch of sciatic nerve were found to be $0.947,0.908$ and 0.956 16 respectively on the non-painful side and $0.991,0.985$ and 0.992 respectively on the 17 painful side.

18 During the forward bending movement, both the total and normalised tibial nerve 19 movements in the longitudinal, axial and hypotenuse planes were significantly reduced 20 in the painful in the FBSS group when compared to the non-FBSS group (Table 2, $\mathrm{P}<$

$210.05)$. There were also significant reduction in the total and normalised tibial nerve 22 movements in the non-painful side in the FBSS group when compared to the non-FBSS 23 group (Table 2, $\mathrm{P}<0.05$ ). 
1 Within the non-FBSS group, there were no significant differences in the total and

2 normalised tibial nerve movement between painful and non-painful side during forward 3 bending (Table $2, \mathrm{P}>0.05$ ).

4 Within the FBSS group, the total and normalised movements of the tibial nerve were 5 significantly reduced in the painful leg when compared to the non-painful leg (Table 2, $6 \quad \mathrm{P}<0.05)$ during the limited forward bending movement of both the lumbar spine and 7 hips (Lumbar flexion: $26.6^{\circ} \pm 5.3^{\circ}$; Hip flexion: $17.2^{\circ} \pm 4.2^{\circ}$ ). 


\section{Discussion}

2 Despite anatomically successful lumbar decompression surgery, FBSS is estimated to

3 occur in $20.8 \%$ of patients within 2 years of surgery[1] and is a significant problem to

4 patients, healthcare providers and society. Further understanding of neural

5 pathomechanics and any involvement in FBSS will potentially contribute to the

6 development of an appropriate intervention for this problematic condition.

7 Consequently, this study examined nerve pathomechanics in two groups of post-lumbar

8 surgical patients; one group with successful clinical outcome (non-FBSS) and the other

9 group with FBSS presenting with persistent leg pain following successful anatomical

10 decompression. Tibial nerve movement during forward bending was compared

11 between the two patient groups, and between the painful and non-painful leg in the

12 FBSS patients, using the previously validated ultrasound imaging technique. This is the

13 first study involving non-invasive in-vivo measures of the magnitude or timing of strain

14 occurring in the tibial nerve during spinal and hip movements in FBSS and non-FBSS

15 patients following lumbar decompression.

17 The observed total movement of the tibial nerve in non-FBSS patients is consistent with

18 the requirement of the nerve tract to accommodate increases in the nerve bed length

19 evoked by hip and lumbar spine flexion during forward bending identified in previous

20 studies[12]. In the FBSS group, the reduced total and normalised tibial nerve excursion

21 could potentially cause increased nerve tension leading to loss of function and pain[9],

22 as demonstrated in the results of this study. Nerve root restrictions can lead to distal

23 alterations in sciatic nerve movement and strain during forward bending as this section

24 of the nerve tract is forced to accommodate more changes in nerve bed length, a finding

25 observed in animal and cadaveric studies[20]. However, it could be expected that the 
1 elimination of nerve root impingement following decompression lumbar surgery would

2 result in the return of normal peripheral nerve movement. All patients with remaining

3 residual pain had a post-operative MRI which failed to identify any remaining

4 restrictions of the nerve root in the lumbar spine. Consequently, it is hypothesised that

5 the persistent postoperative leg pain could be caused by tightening and/or shortening of

6 the sciatic/tibial nerve due to prolonged movement restrictions prior to surgery. If the

7 persistent pain is at least partly due to decreased nerve movement, this could potentially

8 be resolvable with appropriate post-surgical treatment. Persistent post-operative

9 neuropathic pain could also be caused by initial nerve damage, which could lead to

10 permanent neural symptoms with minimal improvement of symptoms expected.

13 People with failed back surgery syndrome have been reported to suffer for an average 14 of 4.7 years[21]. Prolonged nerve root compression has been shown to cause 15 inflammatory changes to the nerve fibres that can result in perineural scarring, nerve 16 fibrosis and intraneural oedema[22]. These changes can result in shortening and 17 tightening of the nerve which subsequently affect neural biomechanical properties[23], 18 leading to mechanosensitivity symptoms including a painful response to nerve stretch 19 during joint movements[10]. Following successful lumbar decompression surgery, it is 20 hoped that normal nerve mechanics will be restored, however, in some instances it 21 appears that this fails to happen. It is proposed that persistent altered nerve mechanics 22 could be responsible for the limited range of motion observed at the lumbar spine and 23 hip in the FBSS patients, which subsequently lead to other nerve changes that produce 24 long term neuropathic pain. Within the FBSS group, both the total and normalised tibial 25 nerve movement was significantly reduced in the painful leg compared with the non- 
1 painful leg at the same limited forward bend position. However, the straight leg raise

2 angle of the non-painful leg was negative (greater than 65 degrees) indicating there was

3 no movement restriction of the sciatic/tibial nerve in the non-painful leg, implying that

4 the limiting factor of the lumbar spine and hip movement was the altered nerve

5 mechanics in the painful leg. A decrease in the ability of peripheral nerves to bend,

6 stretch and glide may limit the transmission of electrical impulses[8] and the dispersion

7 of intraneural fluid[24]. Such limited nerve movement may cause the persistent leg pain

8 in the FBSS group as observed in this study.

9

10 It is well recognised that biological, psychological and social factors can all be

11 influential in the development of low back pain with non-specific low back pain defined

12 as low back pain that cannot be attributed to a specific pathology [25]. Psychological

13 interventions have been shown to be beneficial in the management of idiopathic chronic

14 low back pain in patients who do not have surgically remediable pathology. The

15 Distress and Risk Assessment Method (DRAM) has been shown to be an effective tool

16 for identifying patients that will benefit from psychological intervention[16]. Both

17 patient groups in this study demonstrated no significant differences in both Zung Self-

18 Rating Depression Scale and Modified Somatic Perception Questionnaire which are

19 part of DRAM assessment suggesting that the FBSS patients with chronic persistent

20 postoperative leg pain of this study were not indicated for any psychological

21 interventions. This implies that the persistent leg pain of the FBSS patient group was

22 unlikely to be associated with psychosocial factors but rather due to a mechanical cause

23 such as altered nerve biomechanics. Further research could investigate potential causes

24 of the observed restricted neural mobility including the influence of pain, and whether

25 removal of pain via a spinal nerve block may result in improved neural mobility in 
1 patients with persistent leg pain of FBSS. Further research could also investigate the

2 efficacy of intervention, such as nerve mobilisation in this particular group of patients

3 with failed back surgery syndrome and limited nerve mobility.

5 The limitation of this study was that the assessor was not blinded to the grouping of the

6 participants during the clinical assessments and ultrasound recordings as it was obvious

7 that the participants in the FBSS group with persistent leg pain would normally present

8 with a more limited lumbar and hip movement and limited straight leg raise angle.

9 However, the researcher was blinded to each participant's information or grouping

10 during offline data analysis of the nerve movement data and spinal and hip movement

11 analysis. Participants were also only assessed on one occasion, three to nine-months

12 post-surgery. It was unknown if the participants in the FBSS group with persistent leg

13 pain would have improved their clinical and biomechanical outcome measures if 14 reassessed over time.

\section{Conclusion}

17 This is the first study to compare the tibial nerve mobility evoked by forward bending 18 in patients with and without failed back surgery syndrome following anatomically 19 successful lumbar decompression and demonstrated no psychological stress.

21 Both total and normalised tibial nerve movements were significantly reduced in FBSS 22 patients with persistent leg pain compared with non-FBSS patients following 23 anatomically successful lumbar decompression surgery. In addition, the total and 24 normalised tibial nerve excursion were also more significantly reduced in the painful 25 leg when compared to the non-painful leg in FBSS patients with persistent leg pain. 
1 Further research could investigate the efficacy of intervention, such as nerve

2 mobilisation in this particular group of patients with failed back surgery syndrome and 3 limited nerve mobility.

4

5

6

7 
1 References:

2

3 1. Weir S, Samnaliev M, Kuo TC, Ni Choitir C, Tierney TS, Cumming D, Bruce J,

4 Manca A, Taylor RS, Eldabe S (2017) The incidence and healthcare costs of

5 persistent postoperative pain following lumbar spine surgery in the UK: a cohort

6 study using the Clinical Practice Research Datalink (CPRD) and Hospital Episode

7 Statistics (HES). BMJ open 7(9):e017585. 10.1136/bmjopen-2017-017585

8 2. Suthar P, Patel R, Mehta C, Patel N (2015) MRI evaluation of lumbar disc

9 degenerative disease. J Clin Diagn Res 9(4):TC04-09.

$10 \quad 10.7860 / \mathrm{JCDR} / 2015 / 11927.5761$

11 3. Lurie JD, Birkmeyer NJ, Weinstein JN (2003) Rates of advanced spinal imaging

12 and spine surgery. Spine 28(6):616-620. 10.1097/01.BRS.0000049927.37696.DC

13 4. Eldabe S, Kumar K, Buchser E, Taylor RS (2010) An analysis of the components

14 of pain, function, and health-related quality of life in patients with failed back

15 surgery syndrome treated with spinal cord stimulation or conventional medical

16 management. Neuromodulation 13(3):201-209. 10.1111/j.1525-1403.2009.00271.x

17 5. Fokter SK, Yerby SA (2006) Patient-based outcomes for the operative treatment of

18 degenerative lumbar spinal stenosis. Eur Spine J 15(11):1661-1669.

$19 \quad 10.1007 / \mathrm{s} 00586-005-0033-4$

20 6. Asch HL, Lewis PJ, Moreland DB, Egnatchik JG, Yu YJ, Clabeaux DE, Hyland

21 AH (2002) Prospective multiple outcomes study of outpatient lumbar

22 microdiscectomy: should 75 to $80 \%$ success rates be the norm? J Neurosurg $96(1$

23 Suppl):34-44. 
1 7. Dewing CB, Provencher MT, Riffenburgh RH, Kerr S, Manos RE (2008) The

2 outcomes of lumbar microdiscectomy in a young, active population: correlation by

3 herniation type and level. Spine 33(1):33-38. 10.1097/BRS.0b013e31815e3a42

4 8. Ellis RF, Hing WA (2008) Neural mobilization: a systematic review of randomized

5 controlled trials with an analysis of therapeutic efficacy. J Man Manip Ther

$6 \quad 16(1): 8-22.10 .1179 / 106698108790818594$

7 9. Millesi H, Zoch G, Rath T (1990) The gliding apparatus of peripheral nerve and its

8 clinical significance. Ann Chir Main Memb Super 9(2):87-97.

9 10. Dilley A, Lynn B, Pang SJ (2005) Pressure and stretch mechanosensitivity of

10 peripheral nerve fibres following local inflammation of the nerve trunk. Pain

$11 \quad$ 117(3):462-472. 10.1016/j.pain.2005.08.018

12 11. Yayama T, Kobayashi S, Nakanishi Y, Uchida K, Kokubo Y, Miyazaki T, Takeno

13 K, Awara K, Mwaka ES, Iwamoto Y, Baba H (2010) Effects of graded mechanical

14 compression of rabbit sciatic nerve on nerve blood flow and electrophysiological

15 properties. J Clin Neurosci 17(4):501-505. 10.1016/j.jocn.2009.07.110

16 12. Shum GL, Attenborough AS, Marsden JF, Hough AD (2013) Tibial nerve

17 excursion during lumbar spine and hip flexion measured with diagnostic

18 ultrasound. Ultrasound Med Biol 39(5):784-790.

$19 \quad 10.1016 / j . u l t r a s m e d b i o .2012 .11 .023$

20 13. Dworkin RH, Turk DC, Farrar JT, Haythornthwaite JA, Jensen MP, Katz NP,

21 Kerns RD, Stucki G, Allen RR, Bellamy N, Carr DB, Chandler J, Cowan P,

22 Dionne R, Galer BS, Hertz S, Jadad AR, Kramer LD, Manning DC, Martin S,

23 McCormick CG, McDermott MP, McGrath P, Quessy S, Rappaport BA, Robbins

24 W, Robinson JP, Rothman M, Royal MA, Simon L, Stauffer JW, Stein W, Tollett

25 J, Wernicke J, Witter J, Immpact (2005) Core outcome measures for chronic pain 
1 clinical trials: IMMPACT recommendations. Pain 113(1-2):9-19.

$2 \quad$ 10.1016/j.pain.2004.09.012

3 14. Stratford PW, Binkley J, Solomon P, Gill C, Finch E (1994) Assessing change

4 over time in patients with low back pain. Phys Ther 74(6):528-533.

5 15. Shum GL, Crosbie J, Lee RY (2005) Effect of low back pain on the kinematics

6 and joint coordination of the lumbar spine and hip during sit-to-stand and stand-to-

$7 \quad$ sit. Spine 30(17):1998-2004.

8 16. Hobby JL, Lutchman LN, Powell JM, Sharp DJ (2001) The distress and risk

$9 \quad$ assessment method (DRAM). J Bone Joint Surg Br 83(1):19-21.

10 17. Rebain R, Baxter GD, McDonough S (2002) A systematic review of the passive

11 straight leg raising test as a diagnostic aid for low back pain (1989 to 2000). Spine 12 27(17):E388-395.

18. Coppieters MW, Hough AD, Dilley A (2009) Different nerve-gliding exercises

14 induce different magnitudes of median nerve longitudinal excursion: an in vivo study using dynamic ultrasound imaging. J Orthop Sports Phys Ther 39(3):164171. $10.2519 /$ jospt.2009.2913

17 19. Jensen MP, Turner LR, Turner JA, Romano JM (1996) The use of multiple-item

18 scales for pain intensity measurement in chronic pain patients. Pain 67(1):35-40.

19 20. Smith SA, Massie JB, Chesnut R, Garfin SR (1993) Straight leg raising.

20 Anatomical effects on the spinal nerve root without and with fusion. Spine

21 18(8):992-999.

22 21. Thomson S, Jacques L (2009) Demographic characteristics of patients with severe 23 neuropathic pain secondary to failed back surgery syndrome. Pain Pract 9(3):206$24 \quad 215.10 .1111 / \mathrm{j} .1533-2500.2009 .00276 . x$ 
1 22. Lemke A, Penzenstadler C, Ferguson J, Lidinsky D, Hopf R, Bradl M, Redl H,

2 Wolbank S, Hausner T (2017) A novel experimental rat model of peripheral nerve

3 scarring that reliably mimics post-surgical complications and recurring adhesions.

4 Dis Model Mech 10(8):1015-1025. 10.1242/dmm.028852

5 23. Topp KS, Boyd BS (2012) Peripheral nerve: from the microscopic functional unit

6 of the axon to the biomechanically loaded macroscopic structure. J Hand Ther

$7 \quad$ 25(2):142-151; quiz 152. 10.1016/j.jht.2011.09.002

8 24. Gilbert KK, Roger James C, Apte G, Brown C, Sizer PS, Brismee JM, Smith MP

9 (2015) Effects of simulated neural mobilization on fluid movement in cadaveric

10 peripheral nerve sections: implications for the treatment of neuropathic pain and

11 dysfunction. J Man Manip Ther 23(4):219-225.

$12 \quad 10.1179 / 2042618614 Y .0000000094$

13 25. Apeldoorn AT, Bosselaar H, Ostelo RW, Blom-Luberti T, van der Ploeg T, Fritz

14 JM, de Vet HC, van Tulder MW (2012) Identification of patients with chronic low

15 back pain who might benefit from additional psychological assessment. Clin J Pain

$16 \quad 28(1): 23-31.10 .1097 / A J P .0 b 013 \mathrm{e} 31822019 \mathrm{~d} 0$

17 
6

List of tables

Table 1. Subject characteristics

\begin{tabular}{lcc}
\hline & Non-FBSS & $\begin{array}{c}\text { FBSS with } \\
\text { Persistent leg pain }\end{array}$ \\
\hline Age (years) & $55.6 \pm 13.2$ & $54.4 \pm 12.5$ \\
\hline Height $(\mathrm{cm})$ & $169.3 \pm 6.6$ & $169.1 \pm 7.1$ \\
\hline Weight $(\mathrm{kg})$ & $73.7 \pm 12.7$ & $70.8 \pm 9.7$ \\
\hline Post operation days & $146.1 \pm 36.2$ & $145.4 \pm 36$ \\
\hline $\begin{array}{l}\text { Pre operation pain scale (Visual analogue } \\
\text { scale(VAS)) }\end{array}$ & $8.7 \pm 1.6$ & $8.9 \pm 1.1$ \\
\hline Global rating of change scale $(-7$ to +7$)$ & $6.1 \pm 2.1$ & $2.2 \pm 2.1^{\mathrm{a}}$ \\
\hline Severity of back pain $(\mathrm{VAS})$ & $0.3 \pm 0.7$ & $1.8 \pm 3.1^{\mathrm{a}}$ \\
\hline Severity of leg pain $(\mathrm{VAS})$ & $0.8 \pm 1.4$ & $5.9 \pm 1.8^{\mathrm{a}}$ \\
\hline Modified Somatic Perception Questionnaire & $0.8 \pm 1.6$ & $1.2 \pm 2$ \\
\hline Modified Zung depression scale & $6.1 \pm 5.3$ & $5.8 \pm 4.4$ \\
\hline Lumbar flexion during forward bending $\left({ }^{\circ}\right)$ & $71.9 \pm 8.2$ & $26.6 \pm 5.3^{\mathrm{a}}$ \\
\hline${\text { Hip flexion during forward bending }\left({ }^{\circ}\right)}^{\circ}$ & $29.2 \pm 4.7$ & $17.2 \pm 4.2^{\mathrm{a}}$ \\
\hline Straight leg raise angle $\left(\right.$ painful side, $\left.{ }^{\circ}\right)$ & $76.5 \pm 7.0$ & $42.0 \pm 16.2^{\mathrm{a}}$ \\
\hline Straight leg raise angle $\left(\right.$ non-painful side, $\left.{ }^{\circ}\right)$ & $77.6 \pm 5.7$ & $74.5 \pm 12.8$ \\
\hline
\end{tabular}

$9 \quad{ }^{\text {a }} \mathrm{P}<0.05$, significant differences in painful side between Non-FBSS and FBSS group 10 ( $\mathrm{t}$ test).

11 
1 Table 2. Comparison between Non-FBSS group and FBSS group in the painful side

2 of leg pain.

3

\begin{tabular}{lcccc}
\hline & \multicolumn{2}{c}{ Non-FBSS group } & \multicolumn{2}{c}{ FBSS group } \\
\hline & Mean \pm SD & Mean \pm SD & Mean \pm SD & Mean \pm SD \\
\hline $\begin{array}{l}\text { Total Longitudinal tibial } \\
\text { nerve movement (mm) }\end{array}$ & $10.4 \pm 2.1$ & $11.3 \pm 2.0$ & $1.8 \pm 0.7^{\mathrm{a}}$ & $3.8 \pm 1.0^{\mathrm{b}, \mathrm{c}}$ \\
\hline $\begin{array}{l}\text { Total Axial tibial nerve } \\
\text { movement (mm) }\end{array}$ & $4.5 \pm 0.5$ & $3.7 \pm 0.2$ & $0.2 \pm 0.1^{\mathrm{a}}$ & $1.2 \pm 0.1^{\mathrm{b}, \mathrm{c}}$ \\
\hline $\begin{array}{l}\text { Total Hypotenuse tibial nerve } \\
\text { movement }(\mathrm{mm})\end{array}$ & $11.4 \pm 1.9$ & $11.9 \pm 1.8$ & $1.9 \pm 0.7^{\mathrm{a}}$ & $4.1 \pm 0.9^{\mathrm{b}, \mathrm{c}}$ \\
\hline
\end{tabular}

\begin{tabular}{|c|c|c|c|c|}
\hline $\begin{array}{l}\text { Normalized Longitudinal } \\
\text { tibial nerve movement } \\
\left(\mathrm{mm} /{ }^{\circ}\right)\end{array}$ & $0.1022 \pm 0.0208$ & $0.1114 \pm 0.019$ & $0.0411 \pm 0.01075^{\mathrm{a}}$ & $0.0892 \pm 0.023^{\mathrm{b}, \mathrm{c}}$ \\
\hline $\begin{array}{l}\text { Normalized Axial tibial } \\
\text { nerve movement }\left(\mathrm{mm} /{ }^{\circ}\right)\end{array}$ & $0.0441 \pm 0.005$ & $0.0384 \pm 0.0037$ & $0.0043 \pm 0.00502^{\mathrm{a}}$ & $0.0311 \pm 0.011^{\mathrm{b}, \mathrm{c}}$ \\
\hline $\begin{array}{l}\text { Normalized Hypotenuse } \\
\text { tibial nerve movement } \\
\left(\mathrm{mm} /{ }^{\circ}\right)\end{array}$ & $0.1127 \pm 0.0181$ & $0.1186 \pm 0.0178$ & $0.0419 \pm 0.01101^{\mathrm{a}}$ & $0.0946 \pm 0.0228^{b, c}$ \\
\hline \multicolumn{5}{|l|}{4} \\
\hline \multirow{2}{*}{\multicolumn{5}{|c|}{$\begin{array}{l}{ }^{a} \mathrm{P}<0.05 \text {, significant differences in painful side between Non-FBSS and FBSS group } \\
\text { ( } \mathrm{t} \text { test). }\end{array}$}} \\
\hline & & & & \\
\hline \multicolumn{5}{|c|}{$7{ }^{\mathrm{b}} \mathrm{P}<0.05$, significant differences in the non-painful side between Non-FBSS and } \\
\hline \multicolumn{5}{|l|}{ FBSS group ( $\mathrm{t}$ test $)$} \\
\hline \multicolumn{5}{|c|}{${ }^{\mathrm{c}} \mathrm{P}<0.05$, significant differences between painful and non-painful side within the } \\
\hline \multicolumn{5}{|c|}{$10 \quad$ FBSS group (paired t-test). } \\
\hline
\end{tabular}

